\title{
Late Follow-Up of Rastelli Operation After One-Stage Repair with Right Ventricular Remodeling
}

Miguel Maluf*

\author{
Full Professor, Federal University of São Paulo, Al dos Anapurus, 1580, Ap. 73. Moema. CEP: 04087-005. São Paulo, Brazil
}

\begin{abstract}
Correspondence to: Miguel Maluf, Full Professor, Federal University of São Paulo, Al dos Anapurus, 1580, Ap. 73. Moema. CEP: 04087-005. São Paulo, Brazil Received date: February 22, 2021; Accepted date: March 5, 2021; Published date: March 13, 2021

Citation: Maluf M (2021) Late Follow-Up of Rastelli Operation After One-Stage Repair with Right Ventricular Remodeling. J Med Res Surg 2(2): pp. 1-4.

Copyright: (C2021 Maluf M. This is an open-access article distributed under the terms of the Creative Commons Attribution License, which permits unrestricted use, distribution and reproduction in any medium, provided the original author and source are credited.
\end{abstract}

\section{ABSTRACT}

We report long-term outcomes after one-stage, surgical repair, at an 11-year-old, girl with pulmonary atresia with a ventricular septal defect and anomalous origin of the left coronary artery from the right coronary artery, crossing the right ventricular outflow tract. The operation was carried out with the remodeling of the right ventricle, using a swine valved conduit prosthesis $(22 \mathrm{~mm})$. Twenty-nine years later, the patient is in excellent clinical condition, CF I (NYHA), with normal peripheral oxygen saturation. Recent invasive and not invasive imaging show: the absence of intracardiac shunt, despite presenting prosthesis calcification and signs of mild double lesion of the prosthetic valve. The pulmonary flow is directed uniformly for both lungs. Patients undergoing the implantation of valved conduits during childhood, present mismatch, after a few years of follow-up, and are required to undergo frequent reoperations. In this patient, the indication of a Blalock-Taussig shunt, during childhood, allowed to postpone the surgery and implant a valved conduit with a larger diameter, achieving a long follow-up with a single prosthetic implant.

\section{Keywords:}

Ventricular remodeling; Pulmonary valve; Pulmonary stenosis, Valved conduit, Coronary anomaly

\section{Introduction}

Pulmonary Atresia (PA) with Ventricular Septal Defect (VSD) represents $2.5-3.4 \%$ of all congenital heart defects. Pulmonary Atresia with Ventricular Septal Defect (PA-VSD) is a cyanotic congenital heart disease characterized by underdevelopment of the Right Ventricular (RV) outflow tract, with atresia of the pulmonary valve, a large Ventricular Septal Defect (VSD), and overriding of the aorta.

The classification of anatomical variations of AP w/VSD was proposed by Barbero-Marcial [1]. This classification is based on the presence or absence of native pulmonary arteries (PAs) and the presence or absence of main pulmonary collateral arteries, in Type A, B, C.

Type A: This is the case with this patient, all the bronchopulmonary segments are connected to the central Pas. The pulmonary arteries are confluent and have all pulmonary segments, which are connected to the aortic isthmus by the patent arterial ductus. The initial surgical objective consists of ensuring the presence of PAs adequate in size, distribution, and peripheral resistance to ultimately permit the possibility of total correction.

Right ventricular remodeling, in the AP with VSD, consists of connecting the RV to the AP using a valved conduit, a surgical procedure described, 5 decades ago by Rastelli et al. in 1969 $[2,3]$. This technique has been considered the procedure of choice for the surgical repair of dextro-transposition of the great arteries (D-TGA) associated with Ventricular Septal Defect (VSD) and Left Ventricular Outflow Tract Obstruction (LVOTO). In the original description, the procedure involved the connection of the right ventricle to the pulmonary arteries with a homograft conduit. Its use was also expanded to other anatomic variants as PA w/VSD.

\section{Case presentation}

A 40-year-old (born in 1980), black woman, height: 1.60m, weight: $51 \mathrm{~kg}, \mathrm{BMI}: 15.9$, returns for annual control to ours Cardiovascular Ambulatory.

The diagnosis of AP/VSD associated with anomalous origin of the left coronary artery from the ostium of the right coronary, made in childhood, changed the strategy of surgical approach, about:

- $\quad$ Right ventricular remodeling technique and

- Age at which the corrective operation would be performed Palliative surgical technique

In 1982, at the age of 2 years, a Blalock-Taussig shunt was performed, to improve the pulmonary flow and postpone the definitive correction.

\section{Corrective surgical technique}

The patient was submitted to PA/VSD correction, in 1991, at the age of 11 years by the Rastelli technique. Since 1990, our group has been using pig-valved pulmonary ducts. Manufacture of this prosthesis employs:

- Pulmonary Artery (PA) segment with its valve and

- $\quad$ PA segment without its valve. The anastomosis of the 2 segments is done by coupling, with double suture (Figure 1) [1].

The surgical correction technique was guided by the course of the left coronary artery, originating in the right coronary ostium and crossing the Right Ventricular Outflow Tract (RVOT), near to the origin of the pulmonary trunk. Through a minimal ventriculotomy in RVOT, the VSD was closed, tuning the Left Ventricle (LV) to the Aorta (Ao). Finally, a $22 \mathrm{~mm}$ swine valved conduit was installed between the PA and RV (Figure 2). 


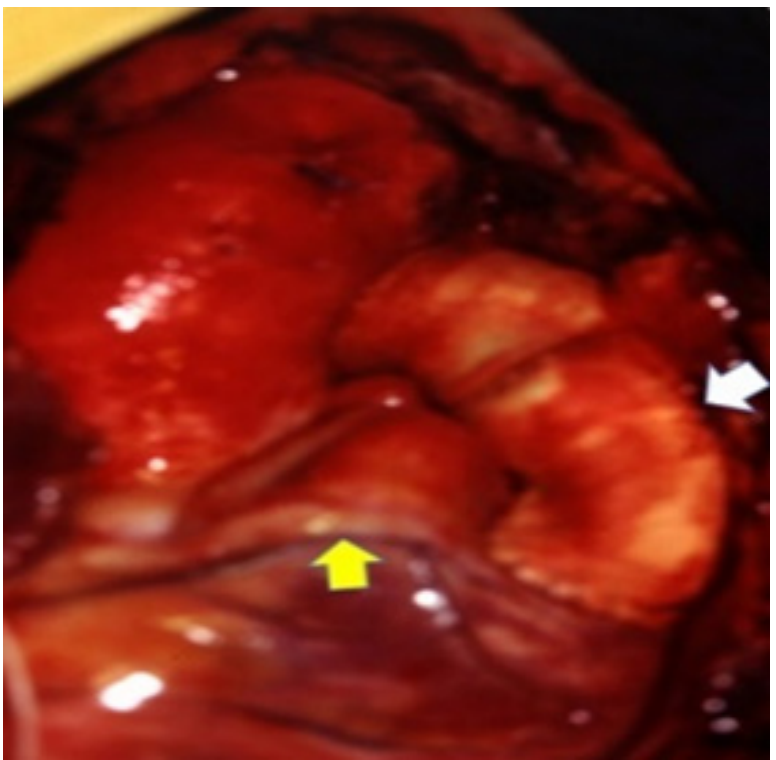

Figure 1: Surgical Photography: Swine valved conduit implanted (white arrow) between the right ventricle and pulmonary artery. Anomalous origin of the left coronary artery, crossing right ventricle outflow tract (yellow arrow).

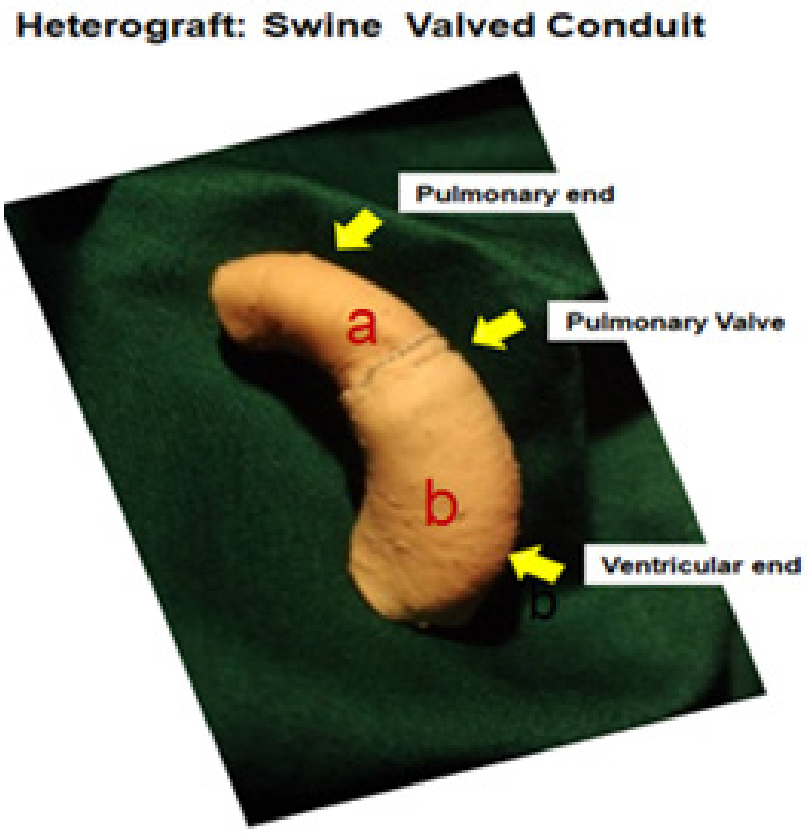

Figure 2: Photography of Swine Valved conduit: Manufacture of this prosthesis employs, a): Pulmonary Artery (PA) segment with its valve and $\mathbf{b}$ ): PA segment without its valve. The anastomosis of the 2 segments is done by coupling, with a double suture.

\section{Clinical Examination}

On physical examination, the patient has a good general condition and normal weight-bearing development and her percent SO2=97\%.

Cardiovascular system examination: A rude systolic and mild diastolic murmur was heard in pulmonary focus. Following the postoperative control protocol, the patient underwent noninvasive and invasive imaging exams to assess hemodynamic and cardiopulmonary functional performance.

\section{Investigations}

Chest X-Ray: Cardiothoracic index $=60 \%$, enlargement of the upper mediastinum, regarding elongation of the pulmonary silhouette, with evident signs of calcification of the valved conduit; Lungs with normal parenchyma and circulation.

ECG: Sinus rhythm, HR: $75 \mathrm{~b} \times$ min, QRS axis: $90^{\circ}$, right bundle branch block.

Transthoracic echocardiogram: Hypertrophic and dilated right ventricle $(30 \mathrm{~mm})$. Preserved biventricular systolic function. Absence of flow through the native pulmonary valve (pulmonary atresia)

Flow accelerated by the valved conduit, determining a peak gradient of $50 \mathrm{mmHg}$ at the level of the prosthetic valve and presents mild pulmonary insufficiency.

Holter study: Sinus rhythm, without pauses, right bundle branch block, HR:69 to $136 \mathrm{~b} \times \mathrm{min}$, and rare extrasystole.

Ergoespirometry test was performed to assess the patient's cardiopulmonary capacity.

Result: Moderately reduced aerobic capacity. Reduced predicted LA/VO2 ratio. Reduced $\mathrm{O} 2$ pulse and tachycardic HR pattern. Physiological BP response. Reduced ventilatory reserve. Excessive ventilatory response to metabolic demand. PETCO2 with reduced values at rest and exercise. Reduction of aerobic capacity due to cardiocirculatory and ventilatory limitations

Angio CT: Increase of the right cavities: RA, RV. Coronary arteries with a single origin from the anterior right coronary sinus. Aortic root $=32 \times 30 \mathrm{~mm}$. Ascending Aortic $38 \times 37 \mathrm{~mm}$. Art. Carotid Dir and Art. Subclavian D born independent and retro tracheal route. RV-TP conduit, calcified with irregularities in the conduit valve segment. The diameter of the Conduit: $20 \times$ $17 \mathrm{~mm} / 15 \times 15 \mathrm{~mm} / 15 \times 10 \mathrm{~mm}$, to the fullest extent. RPA=21 $\times 16 \mathrm{mmm}$. LPA $=21 \times 20 \mathrm{~mm}$ (Figure 3).
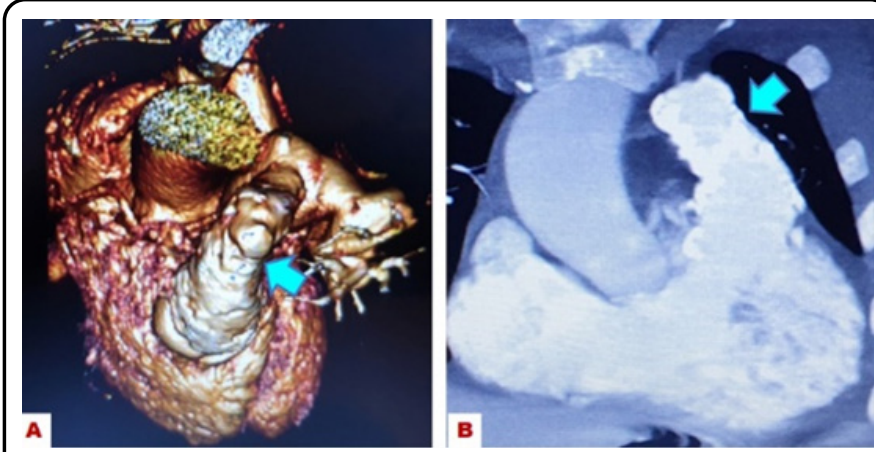

Figure 3: Photography of Angio CT from the heart, A): Image of the swine valved conduit (arrow), B): Contrast image of the right ventricle and valved conduit, with signs of calcification and thickening of the truncal valve (arrow)

Nuclear Magnetic Resonance: Left and right ventricle segmental and global contractility preserved. Calculation of indexed volumes RVFDV $=71.0 \mathrm{ml} / \mathrm{m} 2 ; \mathrm{RVFSV}=24.6 \mathrm{ml} / \mathrm{m} 2$ $\mathrm{RVEF}=65.8 \%$ (Figure 4).

Cardiac catheterization: The hemodynamic study of the right cavities showed:

Moderate stenosis of the conduit. Mild insufficiency of the truncal valve. $\mathrm{RV}=95 \times 05 \mathrm{mmHg}$; Conduit $=61 \times 14(32) \mathrm{mmHg}$; $\mathrm{PA}=60 \times 10$ (30) mmHg; LV=160 × $5 \mathrm{mmHg} ; \mathrm{Ao}=152 \times 82(95)$ $\mathrm{mmHg}$. 


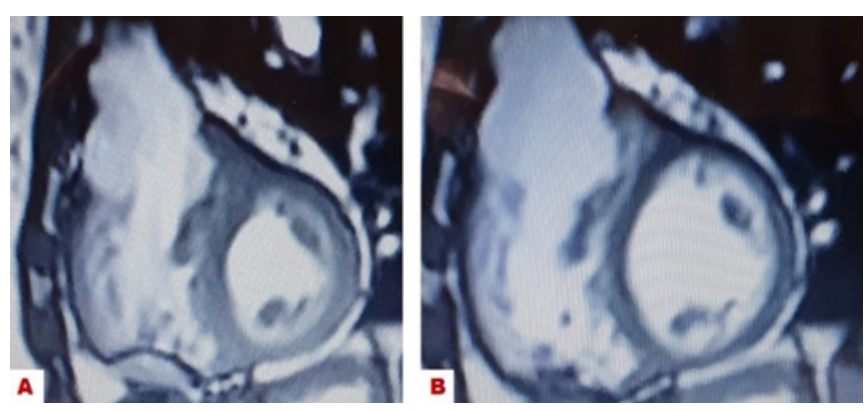

Figure 4: Photography of Magnetic Nuclear Resonance of the heart, A): Contrast image of the right ventricle in systole and $\mathbf{B}$ ): in diastole.

\section{Discussion and Conclusion}

This case report describes a long and large follow-up of patients after Rastelli operations; other similar cases have been published in a series of patients, with different diagnoses, but with the same technique [5-8].

The Mayo Clinic was the pioneer Institution in implementing this technique. From the first case on July 26, 1968, through January 1, 2000, 231 patients have undergone the Rastelli operation at this institution. To examine the late results (mean follow-up greater than 10 years) after this procedure, retrospectively reviewed the 160 early survivors of the Rastelli operation from 19685 In this series of patients published, $41.8 \%$ were between 5 and 9 years old and $34.4 \%$ between 10 and 19 years old. A total of 189 palliative operations had been performed previously, in 123 patients (76.9\%)

It is important to highlight, in this series of patients, the high incidence of palliative surgery performed $(76.9 \%)$ and also the age range of operated patients (76.2\% between 5 and 19 years of age). These were the same criteria used for our patient's surgical approach. Respecting these criteria, it allows the surgeon to implant valved conduits of larger diameter, avoiding mismatch and a greater number of reoperations, to replace the prosthesis.

Regarding the type of conduits implanted by the Mayo Clinic group, varied according to the time interval in which the operation was performed and the preference of the surgeon. An appropriately sized valved conduit is chosen. Cryopreserved homografts are preferred for infants; homografts or porcinevalved Dacron conduits (Medtronic, Inc, Minneapolis, MN) are preferred for older children and porcine-valved conduits are preferred for adults [9-12].

Aware of publication data and disappointed with the results of the conduits' performance, until late follow-up, in 1990 we developed a new heterograft: swine valved conduits, with 2 segments of swine pulmonary artery (Figure 1), indicated for congenital heart disease with discontinuity RV-PA connection. This new prosthesis was standardized to be implanted by, all the surgeons of our group. In the period from 1990 to 2017, more than 100 patients were implanted.

The recommendations of the Mayo Clinic, regarding the technique of conduit implantation, highlight: The valvebearing segment of a valved conduit is placed close to the distal anastomosis to minimize compression of the heart and coronary arteries by the valve when the sternotomy is closed.
It is important to highlight that our $22 \mathrm{~mm}$ diameter swine valved conduit had a perfect adaptation in the remodeling of the RV, despite the presence of the anomalous origin of the left coronary artery of the right coronary ostium, crossing the RV outlet tract.

Data published by the Mayo Clinic, show that: the diameter of the conduits ranged from $10 \mathrm{~mm}$ to $30 \mathrm{~mm}$. The mean diameter was $21.3 \pm 3.0 \mathrm{~mm}$ for the homograft and $22.4 \pm 2.8 \mathrm{~mm}$ for the prosthetic conduits.

The conduct of our group was to implant valved conduits from 18 to $25 \mathrm{~mm}$, to avoid early mismatch. In patients under 5 years of age, the remodeling of the RV was done with a bicuspid swine prosthesis, allowing the growth of the cardiac structures together with the patient [13].

The mean follow-up of the 160 hospital survivors was $11.9 \pm$ 6.9 years (maximum, 25.5 years). 53 known late deaths were ranging. There was no significant difference in survival between age groups 15 years after the operation

Survivorship free of any late reoperation or intervention was $69 \%$ at 5 years, $33 \%$ at 10 years, $19 \%$ at 15 years, and $18 \%$ at 20 years.

The follow-up of our patient is 29 years old (1991-2020). Recent studies have shown good biventricular performance. The RV presents hypertrophy and slight dilation and normal systolic and diastolic volumes. The prosthesis presents calcification of its wall, in all its extension, without compromising RV and PA anastomoses and the prosthetic valve presents fibrosis with moderate double valve lesion, with RV-PA gradient between 36 to $50 \mathrm{mmHg}$.

The assessment of cardiopulmonary performance showed: Reduction of aerobic capacity due to cardiocirculatory and ventilatory limitations

Mayo Clinic's interpretation of conduit failure was defined as the need for reoperation for conduit obstruction (stenosis or extrinsic compression), conduit valve regurgitation, or anastomotic dehiscence. There was conduit failure in 107, $72.8 \%$ patients. Of those patients requiring reoperation for conduit failure in this series, all but two had conduit obstruction; two had predominant conduit regurgitation, and none had anastomotic dehiscence.

According to evolution and data obtained by our patient's invasive and non-invasive exams, a minimally invasive procedure was determined, using Transcatheter Pulmonary Valve Replacement (TPVR) in an elective way.

Mayo Clinic survivorship free of reoperation for conduit failure for the initial conduit placed at the time of the Rastelli operation was stratified according to age at operation. This was worst for the group 0 to 2 years and intermediate for the group 3 to 19 years. Patients 20 to 44 years had a significantly improved survivorship free of reoperation compared with the 0 to 2-year group ( $P=0.002)$ and the 3 to 19-year group $(P=0.02)$. Of the 107 late survivors, New York Heart Association functional class was available for 103 . There were 73 patients $(70.9 \%)$ in functional class I, $25(24.3 \%)$ in class II, and 5 (4.9\%) in class III.

Our patient is in functional class I (NYHA), performs domestic and professional activities, without restriction, and had no major event in her long late follow-up. Medication: B-blockers. 
Mayo Clinic Group comments say: The primary late problem related to the Rastelli operation is the need for conduit replacement because of somatic growth of the patient or to progressive deterioration and calcification of the conduit. The need for conduit replacement was by far the most common reason for reoperation.

Our group's decision-making criteria, with the implant in this first patient of a new swine valved conduits, in 1991, respecting the patient's age (11 years old) and prosthesis diameter $(22 \mathrm{~mm})$, allowed a long late follow-up (29 years), without the need to replace the prosthesis and without events related to the prosthesis. This result allows us to conclude that the adopted conduct was correct and that we must continue, without changes, these criteria that will reduce reoperations and will give a better and higher quality of life to our patients.

\section{References}

1. Barbero-Marcial M, Jatene AD (1990) Surgical management of the anomalies of the pulmonary arteries in the tetralogy of Fallot with pulmonary atresia. Semin Thorac Cardiovasc Surg 2(1): pp. 93-107.

2. Rastelli GC (1969) A new approach to "anatomic" repair of transposition of the great arteries. Mayo Clin Proc 44(): pp. 1-12.

3. Rastelli GC, Wallace RB, Ongley PA (1969) Complete repair of transposition of the great arteries with pulmonary stenosis: $A$ review and report of a case corrected by using a new surgical technique. Circulation 39(2): pp. 83-95.

4. Maluf MA (1991) Reconstruction of the right ventricular outflow tract with swine pulmonary bicuspid or valved conduit prosthesis: An experimental study in sheep. Doctoral thesis. Federal University of São Paulo.

5. Dearani JA, Danielson GK, Puga FJ, et al. (2001) Late Results of the Rastelli Operation for Transposition of the Great Arteries.
Semin Thorac Cardiovasc Surg 4(1): pp. 3-5.

6. Christian Kreutzer, Julie De Vive, Guido Oppido, et al. (2000) Twenty-five-year experience with Rastelli repair for transposition of the great arteries. J Thorac Cardiovasc Surg 120(2): pp. 211-223.

7. Moulton AL, de Leval MR, Macartney FJ, et al. (1981) Rastelli procedure for transposition of the great arteries, ventricular septal defect and left ventricular outflow tract obstruction: Early and late results in 41 cases(1971-1978). Br Heart J45(1): pp. 20-28.

8. Kececioglu D, Vogt J, de Vivie ER, et al. (1988) Late results following the Rastelli corrective operation for transposition of the great arteries. Z Kardiol 77(7): pp. 432-435.

9. Wallace RB, Rastelli GC, Ongley PA, et al. (1969) Complete repair of truncus arteriosus defects. J Thorac Cardiovasc Surg 57(1): pp. 95-107.

10. Malm j R, Bowman FO Jr, Harris PD, et al. (1967) An evaluation of aortic homografts sterilized by electron beam energy. J Thorac Cardiovasc Surg 54(4): pp. 471-477.

11. Agarwal KC, Edwards WD, Feldt RH, et al. (1981) Clinicopathological correlates of obstructed right-sided porcinevalved extracardiac conduits. J Thorac Cardiovasc Surg 81(4): pp. 591-601.

12. Agarwal KC, Edwards WD, Feldt RH, et al. (1982) Pathogenesis of nonobstructive fibrous peels in right-sided porcine-valved extracardiac conduits. J Thorac Cardiovasc Surg 83(2): pp. 584589.

13. Maluf M, Braile DM, Silva C, et al. (2000) Reconstruction of the pulmonary valve and outflow tract with the bicuspid prosthesis in tetralogy of Fallot. Ann Thorac Surg 70(6): pp. 1911-1917. 